\title{
LOS ÑAKIPÁ: GRUPO INDÍGENA EXTINTO DE BAJA CALIFORNIA*
}

\author{
Por \\ Mario Alberto Gerardo Magaña Mancillas**
}

\begin{abstract}
RESUMEN
Buscando ampliar el conocimiento sobre la historia de los grupos indígenas de Baja California, se presenta aquí un acercamiento a uno de ellos que ya no cuenta con representantes o sobrevivientes. En los lejanos años veinte de este siglo, las últimas personas de los f́akipá fueron reconocidas, y después el silencio llenó su recuerdo. Los "que vivían donde se pone el sol", son parte de la historia de este estado, y su rescate es una forma de comprender mejor nuestro pasado. Con el propósito de reconstruir su recuerdo, en este breve ensayo se reune la información básica sobre ellos y se esbozan algunas de sus características.
\end{abstract}

\begin{abstract}
In order to increase the knowledge of the history of the indigenous groups of Baja California, this article approaches one which has no living representatives. The twenties of this century saw the last of the surviving Nakipa; they died, and were forgotten. They "who lived in the place of the setting sun" are a part of the history of this state, and the rescue of what remains to us of them is a means of understanding our past in a better way. So that we may remember them, this brief sketch brings together the basic information we have, and outlines some of their characteristics.
\end{abstract}

La historia de las comunidades indígenas del estado de Baja California prácticamente se encuentra en proceso de integración, falta mucho por realizar en el plano de la síntesis y del esbozo de líneas generales, además son pocos los estudiosos dedicados a este tema y prácticamente no existe un apoyo gubernamental permanente para su desarrollo y difusión. Por lo cual, mi interés en tratar de aportar información y, tal vez, de abrir la discusión sobre la parte histórica de los grupos indígenas de la península, logrando el rescate de fuentes etnohistóricas, históricas, orales y etnograficas dispersas principalmente fuera de Baja California.

* La primera versión de este trabajo fue presentada en el II Simposium Baja California Indígena, realizado por el Instituto cuNA de B.C., A.C., en Ensenada, B.C. (octubre de 1995). ** Licenciado en historia por la Universidad de Guadalajara y maestro en estudios de población con especialidad en historia demográfica por El Colegio de la Frontera Norte. 
De los grupos indígenas que habitan el norte de Baja California, actualmente sobreviven los kumiai (con variantes dialectales como el koatl en Santa Catarina), los paipai, los kiliwa y los cucapá, todos ellos ramas de la familia lingüística yumana que también la integran grupos del sur de California, Arizona y noroeste de Sonora. Sin embargo, éstos no fueron los únicos en la península en tiempos históricos, es decir, en el periodo que va desde el siglo XVI al primer cuarto del XX, ni mucho menos en lo que podríamos denominar la prehistoria que se remonta a unos 10000 u 8000 años de antigüedad.

Entre los grupos indígenas que existieron al norte de la península en los tiempos históricos y que después desaparecieron, se encuentran los ñakipá y los yakakwai, pertenecientes a la citada familia lingüística yumana, y que empezaron a ser identificados por los misioneros dominicos cuando fundaron la misión de Santo Domingo de la Frontera en el último cuarto del siglo XVIII. La última referencia de un miembro identificado como ñakipá y que era hablante de su lengua la encontramos durante la década de los veinte del presente siglo.

Un factor que se debe tomar en cuenta en los estudios sobre los indígenas de Baja California es que su nomenclatura o definición se ha basado en su cercanía a alguna de las misiones dominicas o franciscanas establecidas en el noroeste de Baja California y oeste de California, así se hablo de los "diegueños" por la de San Diego de Alcalá, que en realidad eran los kumiai, o los "domingueños" por la misión de Santo Domingo de la Frontera, que eran los ñakipá. Otra característica que se ha ido acumulando por nuevos estudios, es la definición lingüística, la que ha aportado datos valiosos para la reconstrucción histórica de los indígenas de esta región, sin embargo, continúan siendo parámetros limitados que provienen de sujetos ajenos a las propias culturas indígenas.

Sobre la información que se tiene de los ñakipá durante el asentamiento de las misiones, el fraile Luis Sales anotó que "los de San Fernando y Rosario son humildes, pacíficos y dóciles; los de Santo Domingo y San Vicente son inquietos, soberbios y revoltosos..." En relación con el "Idioma de los Indios", también anotó que "los pueblos de San Francisco de Borja y San Fernando no se distinguen entre sí, en cambio sí se diferencian mucho de los demás; los del Rosario y Santo Domingo son distintos en términos y pronunciación; el Pueblo de San Vicente contiene tres idiomas distintos bajo un mismo gobierno o misión. "El idioma de los Serranos [posiblemente se refiere a los kiliwa y paipai] es muy diferente de todos, y sólo se extiende a la distancia de unas siete leguas, y ya varía..." (Sales, 1960:31 y 62).

Sales aparece en el "Libro de Bautizos" de la misión de Santo Domingo de la Frontera en dos ocasiones presidiendo ceremonias de este tipo, una 
en 1778 y otra en 1779 , lo que indicaría más que estuvo de tránsito por lo menos en esta misión, más que implicara una permanencia por tiempo significativo (AM IIH-UABC, 12:96). Probablemente sus referencias o bases son las opiniones expresadas por los misioneros encargados de casa sitio, más que su propia experiencia.

Fuera de las cartas de Sales, ningún diario o compilación de correspondencia se ha publicado de algún misionero dominico, en general se ha aceptado que ninguno de estos frailes escribió alguna obra de la talla de Miguel del Barco o de Juan Jacobo Baegert, no obstante, mientras no se puedan explorar acervos como el de Saint Albert's College, no se puede tener esa certeza. Existe mucha documentación por recuperar y estudiar, más allá de intentos apologéticos o condenatorios.

En el siglo $\mathrm{xX}$, con el impulso de las ciencias sociales, algunos investigadores de formación académica contienen en sus obras referencias explícitas sobre los ñakipá, destacando varios estudios. En 1959, Homer Aschmann publicó su obra The Central Desert of Baja California: Demography and Ecology, donde se señala que "los ñakipa son poco conocidos y aparentemente extintos, al menos culturalmente. Parece que estuvieron relacionados cercanamente a los Diegueños [entiéndase kumiai], quienes sobreviven en número considerable" (Aschmann, 1987:42). Es de destacar, la diferenciación que hace este autor entre la extinsión física y la cultural, punto que más investigadores deberíamos desarrollar, sobre todo en líneas como el mestizaje y el proceso de reclasificación socioétnica colonial para obtener ventajas o libertades.

En un estudio sobre los kiliwa realizado por Jesús Ángel Ochoa Zazueta, el autor recoge que dentro de las leyendas e historias de este grupo se indica que cerca del cerro kaykesiaí, rumbo al oeste "había mucha gente" y entre éstos estaban los nñak?ipa(i), que según la interpretación de Ochoa Zazueta también eran conocidos como kawi?a o "los recolectores de panales de abeja". En otra parte de este trabajo, donde se relacionan a los vecinos de los kiliwa, se indica que al oeste vivían los ?ipápa(i) (la "gente antigua") y los was-?iá ("los ceramiqueros"), y al sur de éstos y de las montañas de la sierra de San Pedro Mártir estaban los nñaki'pa(i) kawi?a, expresión que traduce como "los recolectores de panales de abeja, los que viven de la miel" (Ochoa Zazueta, 1978:53 y 150). La ubicación geográfica se podría identificar con los actuales valles de Camalú y de San Quintín, donde se ubicaron la misión de Santo Domingo de la Frontera y su visita de San Telmo. Es de señalar por parte de la identificación de Ochoa Zazueta que no establece ninguna definición de ñakipá, ya que en la segunda forma da dos versiones del vocablo kawi?a, ni presenta justificación de la diferencia ortográfica-lingüística de sus dos formas de llamar al grupo que nos ocupa. 
Por su parte: Peter Gerhard en su trabajo The North Frontier of New Spain, $\tan$ sólo hace referencia que dentro de la familia lingüística yumana estaban los idiomas "diegueño" (kumiai), paipai y ñakipá, hablándose más al sur el cochimí en los tiempos prehistóricos e históricos (Gerhard, 1982:288); y el maestro Miguel León-Portilla explica que:

en el área donde se levantaron las misiones del Rosario, Santo Domingo, San Telmo, hasta llegar a la de San Vicente, incluyendo provablemente también, en la sierra, el sitio donde se erigió la misión de San Pedro Mártir, habitaban gentes conocidas con el nombre de ñakipas o yakakwal. El nombre de ñakipa significa "el pueblo del oeste. (León-Portilla, 1983:39, se respeta la ortografía).

Es de anotar que León Portilla incluye dentro del "territorio" ñakipá a la misión de Nuestra Señora del Santísimo Rosario de Viñadaco que generalmente se reconoce que era habitada por indigenas cochimi, y a la de San Pedro Mártir que posiblemente ese espacio era ocupado por miembros del grupo kiliwa o era en todo caso zona de "frontera" entre los kiliwa y ñakipá.

En tiempos históricos, este territorio [en referencia a la parte norte de Baja California] estaba habitado por indígenas de la familia lingüística yumana, quienes se establecieron en dos sitios marcadamente distintos. En el delta del río Colorado se asentaron los kojuana, jalykwamrai, cucapa y algunas bandas paipai. En la montaña y costa encontraron cabida los kamiai, tipai, koal [estos tres se comprenden en el k'miai], kolew, ñakipa y propablemente algunos paipai. (Zárate Loperena, 1987:66).

Ésta es la distribución propuesta por David Zárate Loperena en su artículo "Los pobladores aborígenes de Baja California", que mantiene a grandes razgos la ubicación usualmente aceptada para los grupos indígenas de descendencia yumana, basándose de manera general en los condicionamientos del hábitat que en el momento prehistórico e histórico son importantes tomar en cuenta para comprender mejor su cultura y evolucion.

En un excelente trabajo de síntesis y análisis, Ralph Michelsen aborda la problemática de las definiciones de la territorialidad indígena, en donde en relación con el tema que nos ocupa señala que:

Al ver un mapa del norte de la Baja California, que fuera elaborado con el propósito de delimitar los territorios de los diversos grupos indígenas americanos, uno se encuentra con territorios catalogados como: tipai [entiéndase kumiai], paipai, kiliwa, ñakipa [sic], y así sucesivamente (Michelsen, 1991:151). 
Explícitamente sobre los ñakipá, en cuanto a la definición de su nombre, el autor escribe que "los indios que vivían al suroeste de los kiliwas eran llamados ñakipá ('gente de occidente' o 'de donde se pone el sol'). [Y que] Este mismo grupo era llamado Yak ka kwai ('cosa cruda') por los paipai" (Michelsen, 1991:56).

Como síntesis de este breve repaso, se puede plantear que ios indígenas que habitaron el espacio inmediato y mediato a la misión de Santo Domingo de la Frontera y su visita de San Telmo, a finales del siglo XVIII y principios del xIX, eran diferentes a los que habitaron en la misión de Nuestra Señora del Santísimo Rosario de Viñadaco al sur, y a los del este rumbo a la sierra de San Pedro Mártir, aunque no sabemos hasta este momento, si eran los mismos que habitaban en el área de San Vicente Ferrer. El nombre de este grupo era ñakipá, que es una denominación kiliwa que significarfa como "los que viven hacia donde se pone el sol", aunque León-Portilla y Michelsen señalan que también eran conocidos como yakakwai o yak ka kwai que es una definición desde la perspectiva paipai. Lo que indicaría inicialmente que los ñakipá tenían mayor relación con los kiliwa y los paipai. No obstante, Aschmann indica por su parte que estaban relacionados con los kumiai y no necesariamente con aquellos.

Siguiendo la línea de las aportaciones de los lingüistas, ahora esbocemos lo encontrado en un interesante artículo sobre los ñakipá y los yakakwai que publico Mauricio J.Mixco, titulado "The Linguistic Affiliation of the Nakipa and Yakakwai of Lower California". Aunque este trabajo fue impreso en el año de 1977, la investigación fue realizada en 1968 con base en las anotaciones y cuadernos de campo de J.P. Harrington, que a su vez los había reunido en un viaje científico durante la década de los años veinte por Baja California. Las notas y fotografías que logro reunir este investigador son sumamente valiosas y pueden proporcionar información directa de los grupos indígenas a finales del periodo histórico y de algunos que ya desaparecieron como los ñakipá. Sin embargo, se encuentran en Estados Unidos y es difícil su consulta, obviamente no existe un inventario en español.

Al comparar un vocabulario de la lengua "domingueño" [como la denomina Harrington] o ñakipá, con uno del kiliwa elaborado por el propio Mauricio Mixco, llega a la conclusión inicial de que son "virtualmente idénticos" (Mixco, 1977:191). Esto contradice la postura o conocimiento general producto de las fuentes etnohistóricas de que existía una incomprensión o distanciamiento entre los ñakipá y los kiliwa, e incluso un conflicto entre ambos grupos.

Para Mixco, una hipotesis plausible sería que la aparente dificultad de entendimiento entre los kiliwa y los ñakipá se debió a la influencia sobre 
los últimos de los cochimí, que para el autor fue la primera lengua indígena hablada en la misión de Santo Domingo de la Frontera, núcleo de las fuentes históricas de los ñakipá. Lo que se refuerza con el hecho que el núcleo básico del poblamiento dominico, fue con un grupo de indígenas cochimí principalmente de la misión de San Francisco de Borja (Magaña, 1994). A manera de ejemplo, el primer bautizo de un nacido en la citada misión fue el de Domingo [8 de junio de 1776], "hijo legítimo de Alexo Salazar y Apolonia de la misión de San Borja [..] fue su madrina Bárbara mujer de Jorge indios de San Borja..." (AM IIH UABC, 12:195).

Los ñakipá fueron ingresando a la esfera misional dominica poco a poco, relacionándose con los cochimí conversos y con otros que se trasladaron por razones eclesiástico-administrativas, creando una serie de mestizajes y de uniones intergrupales, con el consecuente intercambio cultural. De ahí que por eso, el fraile Luis Sales encuentra "tres idiomas distintos" en la misión de San Vicente, si tomamos en cuenta que era la sede regional y presidio.

Considero que la hipótesis del enfrentamiento ñakipá-kiliwa es consecuencia de una interpretación de las fuentes históricas, más bien referente a una incomprensión entre los indígenas adscritos a la misión de Santo Domingo de la Frontera, principalmente a inicios del siglo XIX, con influencias cochimi, mestizas e hispanas, con los indígenas que vivían fuera de la influencia misional y que conservaban mayores pautas culturales tradicionales, tanto de los propios ñakipá como de otros grupos. No se puede asegurar que antes de la entrada de los misioneros era la misma circunstancia, al parecer estamos ante un fenómeno producto del periodo misional, más que una característica previa de los grupos indígenas involucrados.

Volviendo al aspecto lingüístico, para Mauricio Mixco la lengua ñakipá es una variante de la kiliwa, como un dialecto de la lengua principal, así podremos hablar de que el ñakipá era una variante dialectal del kiliwa, que con el contacto del cochimí producto de la ingerencia misional sufri 6 algunas modificaciones de tipo fonético, más que de estructura, como se aprecia en sus tablas comparativas más específicas. Esto nos lleva a plantear que los ñakipá eran del grupo kiliwa o muy cercanos, por lo menos poco antes de la entrada misional y el cambio de siglo XVIII al XIX, y que con las influencias ajenas a los indígenas propiamente dichos se fueron alejando culturalmente.

Recordando lo citado por Miguel León-Portilla y Ralph Michelsen, ellos consideran que los ñakipá eran también conocidos como "yakakwai" o "yak ka wai", que el segundo interpreta como "cosa cruda" en lengua paipai. Sobre este particular, Mixco igualmente analiza las anotaciones de Harrington, llegando a la conclusión de que el yakakwai era una variante 
dialectal del paipai, que habitaba la región costera centrada en la misión de San Vicente Ferrer, con una posible influencia de la lengua kumiai (Aschmann señaló que los "ñakipă" estaban relacionados con este grupo), y definitivamente diferente a los ñakipá. Posiblemente la confusión entre los ñakipá y los yakakwai fue debida a su temprana desaparición y a la falta de estudios metódicos sobre la historia indígena de Baja California.

Es de destacar que para indígenas hablantes del grupo paipai, las anotaciones o inventarios de este idioma realizados por Mauricio Mixco contienen algunos puntos discordantes, debido a la forma de captura y comprensión de la fonética indígena del lingüista, $\mathrm{e}$ incluso señalan que podría suceder lo mismo con el kiliwa, creando confusiones al momento de comparar lenguas y realizar las interpretaciones, que no necesariamente reflejan el significado que los hablantes le querían dar. Mientras no se tengan todos los estudios necesarios sobre la historia, cultura, costumbres e idiomas de los indígenas de Baja California, las propuestas no dejan de ser hipótesis de trabajo, valiosas pero superables.

Por último, como balance de este breve ensayo histórico, tenemos que en la parte norte de lo que hoy es el estado de Baja California, sin limitarse a esta jurisdicción, durante el periodo misional dominico (1773-1850) existieron los grupos lingüísticos de la familia yumana de los cucapá, los k'miai, los paipai y los kiliwa. Que estos dos últimos tuvieron a su vez expresiones dialectales: los yakakwai y los ñakipá respectivamente. Que los hablantes del ñakipá habitaron el área inmediata y mediata a la misión de Santo Domingo de la Frontera (fundada en 1775 y abandonada en 1839, pero que continuó siendo visitada por los misioneros hasta 1850 ), que estaban relacionados con los kiliwa, con influencias lingüísticas de los cochimí, por habitar la parte sur de la frontera cultural yumana, y las interacciones forzadas entre los miembros de los grupos indígenas.

La existencia de los ñakipá en una zona dirigida hacia la costa del Pacífico condicionó sus pautas culturales, aunque es posible que antes del contacto misional y limitadamente después de éste, existiera un contacto permanente con los kiliwa de la zona serrana y del golfo, sobre todo en el otoño durante la cosecha del piñón y la bellota. Esto permitió que una parte de la población ñakipá, que decidió alejarse del sistema misional se reincorporara a los kiliwa: "una de las principales explicaciones del despoblamiento de Santo Domingo de la Frontera, se debió a la migración de grupos de indígenas de esta misión hacia territorios usufructuados por indígenas no dominados por los misioneros" (Magaña, 1994:174).

Falta señalar las posibles direcciones de las influencias culturales, que considero necesarias para entender a estos grupos en su tiempo y espacio, y posterior sobrevivencia: los ñakipá como variante dialectal del kiliwa es 
este su sustrato cultural básico, pero con la implantación del sistema misional dominico, los que quedaron en la mision y sus alrededores recibieron, cada vez más, influencia de los cochimí (además de los mestizos e hispanos); los kiliwa mantuvieron una especie de aislamiento, aunque parece que tuvieron algunos contactos con los cochimí: los yakakwai, como el caso de los ñakipá, tienen una base cultural paipai que después fue modificada por influencia kumiai y tal vez cochimí; y los paipai tuvieron contactos con los kumiai, sobre todo en el área de la misión de Santa Catarina.

Tal vez, una posible explicación de la sobrevivencia de los kumiai, paipai, kiliwa y cucapá fue que los dos primeros se replegaron hacia áreas de refugio fuera del sistema misional dominico e inicial colonización civil, centradas en la costa del Pacífico y valles inmediatos, y los dos últimos estaban precisamente en esa condición. Los ñakipá y los yakakwai no sobrevivieron culturalmente porque sus "territorios" fueron ocupados por las misiones, presidios y rancheros, aunque parte de sus integrantes se unieron a los grupos kiliwa y paipai fortaleciendo numéricamente a los mismos. La denominación usada para los indígenas de Baja California de "montañeses", más que reflejar una característica cultural o de adaptación a su medio ambiente, es una pauta de sobrevivencia. 


\section{BIBLIOGRAFIA}

AM IIH-UABC. Acervo de microfilmes del Instituto de Investigaciones Históricas de la UABC.

ASCHMANN, Homer. 1987. The Central Desert of Baja California: Demography and Ecology. Colecc. Ibero-Americana 42. Berkeley: University of California Press.

GERHARD, Peter. 1982. The North Frontier of New Spain. Princeton: Princeton University Press.

LEON-PORTILLA, Miguel. 1983. "Los primeros californios: prehistoria y etnohistoria", en David Piñera Ramírez [coord.] Panorama Historico de Baja California. Tijuana: UABC.

MAGAÑA Mancillas, Mario Alberto Gerardo. 1994. "Santo Domingo de la Frontera. Estudio histórico demográfico de una misión de Baja California: 1775-1850". Tesis de maestraa, El Colegio de la Frontera Norte.

MICHELSEN, Ralph. 1991. "La territorialidad del indígena americano en la tierra alta del norte de la Baja California", en Estudios Fronterizos 24-25 (enero-abril/mayo-agosto). Mexicali: IIS-UABC.

MIXCO, Mauricio J. 1977. "The Linguistic Affiliation of the Ñakipa and Yakakwai of Lower California", en International Journal of American Linguistics 43:3, julio, University of Chicago.

OCHOA Zazueta, Jesús Ángel. 1978. Los kiliwas, y el mundo se hizo así. Serie de Antropología Social 57. México: Instituto Nacional Indigenista.

SALES, Luis. 1960. Noticia de la provincia de California, 1974. Colecc. Chimalistac 6. Madrid: Ediciones José Porrúa Turanzas.

ZÁRATE Loperena, David. 1987. "Los pobladores aborígenes de Baja California", en Memoria del IV Simposium de Historia Regional. Mexicali: Gobierno del Estado de Baja California. 